Review Article

\title{
Small Dense Low-Density Lipoprotein as Biomarker for Atherosclerotic Diseases
}

\author{
Ekaterina A. Ivanova, ${ }^{1}$ Veronika A. Myasoedova, ${ }^{2,3}$ Alexandra A. Melnichenko, ${ }^{2}$ \\ Andrey V. Grechko, ${ }^{4}$ and Alexander N. Orekhov ${ }^{2,5}$ \\ ${ }^{1}$ Department Development and Regeneration, KU Leuven, 3000 Leuven, Belgium \\ ${ }^{2}$ Laboratory of Angiopathology, Institute of General Pathology and Pathophysiology, Moscow 125315, Russia \\ ${ }^{3}$ Centro Cardiologico Monzino IRCCS, 20138 Milan, Italy \\ ${ }^{4}$ Federal Scientific Clinical Center for Resuscitation and Rehabilitation, 14-3 Solyanka Street, Moscow 109240, Russia \\ ${ }^{5}$ Institute for Atherosclerosis Research, Skolkovo Innovation Center, Moscow 121609, Russia
}

Correspondence should be addressed to Veronika A. Myasoedova; veronika.myasoedova@ccfm.it

Received 10 February 2017; Accepted 12 April 2017; Published 9 May 2017

Academic Editor: Manuela Curcio

Copyright (c) 2017 Ekaterina A. Ivanova et al. This is an open access article distributed under the Creative Commons Attribution License, which permits unrestricted use, distribution, and reproduction in any medium, provided the original work is properly cited.

\begin{abstract}
Low-density lipoprotein (LDL) plays a key role in the development and progression of atherosclerosis and cardiovascular disease. LDL consists of several subclasses of particles with different sizes and densities, including large buoyant (lb) and intermediate and small dense (sd) LDLs. It has been well documented that sdLDL has a greater atherogenic potential than that of other LDL subfractions and that sdLDL cholesterol (sdLDL-C) proportion is a better marker for prediction of cardiovascular disease than that of total LDL-C. Circulating sdLDL readily undergoes multiple atherogenic modifications in blood plasma, such as desialylation, glycation, and oxidation, that further increase its atherogenicity. Modified sdLDL is a potent inductor of inflammatory processes associated with cardiovascular disease. Several laboratory methods have been developed for separation of LDL subclasses, and the results obtained by different methods can not be directly compared in most cases. Recently, the development of homogeneous assays facilitated the LDL subfraction analysis making possible large clinical studies evaluating the significance of sdLDL in the development of cardiovascular disease. Further studies are needed to establish guidelines for sdLDL evaluation and correction in clinical practice.
\end{abstract}

\section{Introduction}

High incidence of atherosclerosis and associated cardiovascular diseases (CVD) urges the study of the causes and the risk factors of their development. Atherosclerotic plaque growth is dependent on the uptake of circulating cholesterol by subendothelial cells. Hypercholesterolemia is one of the well-understood risk factors of atherosclerosis, and cholesterol-lowering therapy is widely used in clinical practice for treatment of CVD $[1,2]$. However, the CVD risk reduction achieved in most of the clinical studies was not higher than $30 \%$ indicative of other important risk factors that have to be taken into account [3-5]. A strong line of evidence demonstrates that the development and progression of atherosclerosis are dependent not only and not so much on the amount as on the specific properties of circulating lipoproteins $[6,7]$.

Circulating lipoprotein particles vary in size, density, and lipid and apolipoprotein composition and can be separated into several classes based on physical and chemical parameters. Low-density lipoprotein (LDL) is the major source of atherosclerotic lipid storage, whereas highdensity lipoprotein (HDL) is not atherogenic, and its level inversely correlates with the atherosclerotic CVD risk [8]. Small dense LDL (sdLDL) is especially common in the serum of atherosclerosis patients and is susceptible to chemical modifications that increase their atherogenicity $[9,10]$. The analysis of plasma LDL profile can be performed by ultracentrifugation or gradient gel electrophoresis that can separate the LDL particles based on their density or size 
correspondingly. Other methods have been used to assess the LDL particle size, charge, or chemical properties and will be discussed later in this review. Currently, the development of cheap and reliable LDL profiling methods for routine clinical practice remains a challenging goal.

Numerous clinical studies have been conducted to establish the link between the composition of circulating LDL particles and the risk of atherosclerosis and CVD development. According to the current consensus, 2 main phenotypes, $\mathrm{A}$ and $\mathrm{B}$, are defined based on plasma $\mathrm{LDL}$ profile, with intermediate $A / B$ phenotype lying in between [11]. The phenotype $A$ is characterized by the predominance of large buoyant LDL (lbLDL) and the phenotype B by the predominance of sdLDL $[12,13]$. Phenotype B was reported in a number of diseases, including metabolic disorders $[14,15]$, obesity $[16,17]$, and type 2 diabetes $[18,19]$ and is considered as a risk factor of coronary heart disease (CHD). Moreover, this phenotype was associated with the elevated plasma triglyceride (TG) level, reduced HDL cholesterol (HDL-C), and high-hepatic lipase activity [20]. The predominance of sdLDL is currently accepted as a risk factor for CVD by the National Cholesterol Education Program (NCEPIII) [21]. Apart from density and size, LDL particles may vary in chemical composition because of a series of modifications that they can undergo in human blood. Among them, lipoprotein(a) $(\operatorname{Lp}(\mathrm{a}))$, which contains an additional lipoprotein molecule covalently bound to apolipoprotein $\mathrm{B}$, has been characterized as an additional cardiovascular risk factor [22]. Detection and measurement of modified LDL particles is of special interest, as these types of LDL can be a better marker for increased atherosclerosis, although their content in blood might be scarce in comparison with native LDL.

\section{LDL Subclasses and Methods of Their Identification}

LDL is broadly defined as lipoprotein fraction with density ranging from 1.006 to $1.063 \mathrm{~g} / \mathrm{ml}$, which can be isolated by various laboratory methods. This range also includes the intermediate density lipoprotein (IDL) and very lowdensity lipoprotein (VLDL). More precisely, LDL is known to have a density from 1.019 to $1.063 \mathrm{~g} / \mathrm{ml}$. Ultracentrifugation and gradient gel electrophoresis (GGE) with their modifications are widely used for LDL analysis. In most of the studies using these methods, LDL particles are classified into 3 or 4 subclasses, including large (LDL I), intermediate (LDL II), small (LDL III), and, in some studies, very small (LDL IV) LDLs [3, 18]. LDL III and LDL IV (when discerned) are referred to as sdLDL. However, the classification of LDL based on different analytical methods lacks uniformity, and care should be taken while comparing the results of clinical studies employing different methods.

Historically, the first method that allowed separation of different LDL fractions was analytical ultracentrifugation $[18,23-25]$. In this method, LDL particles are separated based on their flotation rate (Sf). In studies, where three LDL subclasses are defined, LDL I, II, and III have densities of $1.025-1.034 \mathrm{~g} / \mathrm{ml}, 1.034-1.044 \mathrm{~g} / \mathrm{ml}$, and $1.044-1.060 \mathrm{~g} / \mathrm{ml}$, respectively [26, 27]. In some studies, very small LDL IV particles are separated. Phenotype pattern A is characterized by the predominance of LDL I and II and atherogenic phenotype pattern $\mathrm{B}$ by the predominance $(>50 \%)$ of LDL III and IV. Different ultracentrifugation methods result in slight variations in the density of the separated LDL. For instance, iodixanol gradient gives lower densities of LDL particles than traditional salt gradient, because the particles maintain their native hydration $[3,28,29]$.

Another widely used method of LDL subfraction analysis is GGE under nondenaturating conditions. In this method, LDL subclasses are separated by their electrophoretic mobility, which is determined by the size and shape of the lipoprotein [30]. Studies using GGE separation of LDL define 4 subclasses: LDL I (large LDL, peak diameter $26.0-28.5 \mathrm{~nm}$ ), LDL II (intermediate LDL, 25.5-26.4 nm), LDL III A and B (small LDL, 24.2-25.5 nm), and LDL IV A and B (very small LDL, 22.0-24.1 nm) [31]. Two phenotypes can be distinguished based on peak LDL particle diameters: $>25.5 \mathrm{~nm}$ for phenotype pattern A (large and intermediate LDL) and $\leq 25.5 \mathrm{~nm}$ for phenotype pattern B (small and very small LDL). There is a strong correlation between size and density of LDL particles analyzed by ultracentrifugation and GGE, respectively; however, these parameters are not identical. Some authors used tube gel electrophoresis for LDL subfraction analysis for rapid acquisition of quantitative results $[32,33]$.

Nuclear magnetic resonance (NMR) can be employed for studying of lipoprotein classes in blood plasma, including subclasses of LDL. However, the results of particle size measurement by NMR differ significantly from the GGE data in the same patients and can not be directly compared. sdLDL is determined by NMR as particles with sizes from 18.0 to $20.5 \mathrm{~nm}[34,35]$.

Other methods of LDL fraction analysis include high-performance liquid chromatography (HPLC) with gel filtration columns [36], dynamic light scattering [37, 38], ion mobility analysis [39, 40], and homogenous assay analysis [41]. The latter is of special interest because of its high reproducibility and suitability for large-scale clinical trial use. The homogenous assay for detection of sdLDLcholesterol was first described by Hirano et al. [41]. Since then, the assay has been modified to simplify the analytical procedure. In the modified method, sdLDL (particle size $15.0-20.0 \mathrm{~nm})$ is separated from lbLDL using detergent and sphingomyelinase treatment, and sdLDL-cholesterol concentration is measured. The method separates sdLDL fraction with a density from 1.044 to $1.063 \mathrm{~g} / \mathrm{ml}$ using standard clinical laboratory equipment $[42,43]$. The comparison of some of the most widely used methods of LDL subclasses analysis is presented in Table 1.

As the clinical and diagnostic significance of LDL subclasses becomes evident, the standardization problem comes into prominence. Different methods of LDL subclass analysis deliver different results, and significant variations are possible even within one method. It is currently difficult to determine which of the existing approaches can be recommended as the most accurate and, at the same time, suitable for clinical use. Moreover, no data is currently 
TABLE 1: LDL subclasses separated by different laboratory methods.

\begin{tabular}{ccccc}
\hline & & & \multicolumn{2}{c}{ Small dense LDL } \\
\multirow{2}{*}{ Method } & LDL I & LDL II & LDL III & LDL IV \\
& Large & Intermediate & Small & Very small \\
\hline
\end{tabular}

UC density gradient (density)
(a) [44]
(a) $1.019-1.023 \mathrm{~g} / \mathrm{ml}$
(a) $1.023-1.034 \mathrm{~g} / \mathrm{ml}$
(a) $1.034-1.044 \mathrm{~g} / \mathrm{ml}$
(a) $1.044-1.060$
(b) [26]
(b) $1.025-1.034 \mathrm{~g} / \mathrm{ml}$
(b) $1.034-1.044 \mathrm{~g} / \mathrm{ml}$
(b) $1.044-1.060 \mathrm{~g} / \mathrm{ml}$

UC iodixanol gradient (density)
(a) [28]
(a) $1.016-1.028 \mathrm{~g} / \mathrm{ml}$
(b) [29]
(b) $1.022-1.028 \mathrm{~g} / \mathrm{ml}$

(a) $1.028-1.043 \mathrm{~g} / \mathrm{ml}$

(b) $1.028-1.041 \mathrm{~g} / \mathrm{ml}$

GGE (peak diameter)
(a) [30]
(a) $26.35-28.5 \mathrm{~nm}$
(a) $25.75-26.34 \mathrm{~nm}$
(a) $22.0-25.74 \mathrm{~nm}$
(b) $[45]$
(b) $26.0-28.5 \mathrm{~nm}$
(b) $25.5-26.4 \mathrm{~nm}$
(b) $24.7-25.5 \mathrm{~nm}$ (III A)
(b) $23.3-24.1 \mathrm{~nm}(\mathrm{IV} \mathrm{A})$
$24.2-24.6 \mathrm{~nm}$ (III B)
$22.0-23.2 \mathrm{~nm}$ (IV B)
Ion mobility (peak size) [39]
$21.9-23.8 \mathrm{~nm}$
$21.1-21.9 \mathrm{~nm}$
$20.17-21.1 \mathrm{~nm}$
$18.0-20.17 \mathrm{~nm}$

NMR (peak size)
(a) $[34,35]$
(a) $21.3-22.7 \mathrm{~nm}$
(a) $19.8-21.2 \mathrm{~nm}$
(b) [32]
(b) $20.6-22.0 \mathrm{~nm}$
(b) $20.4-20.5 \mathrm{~nm}$

(a) $18.3-19.7 \mathrm{~nm}$

Homogenous assay [42, 43]

(b) $19.0-20.3 \mathrm{~nm}$

Separated particles with density $1.044-1.063 \mathrm{~g} / \mathrm{ml}$ Phenotype pattern B

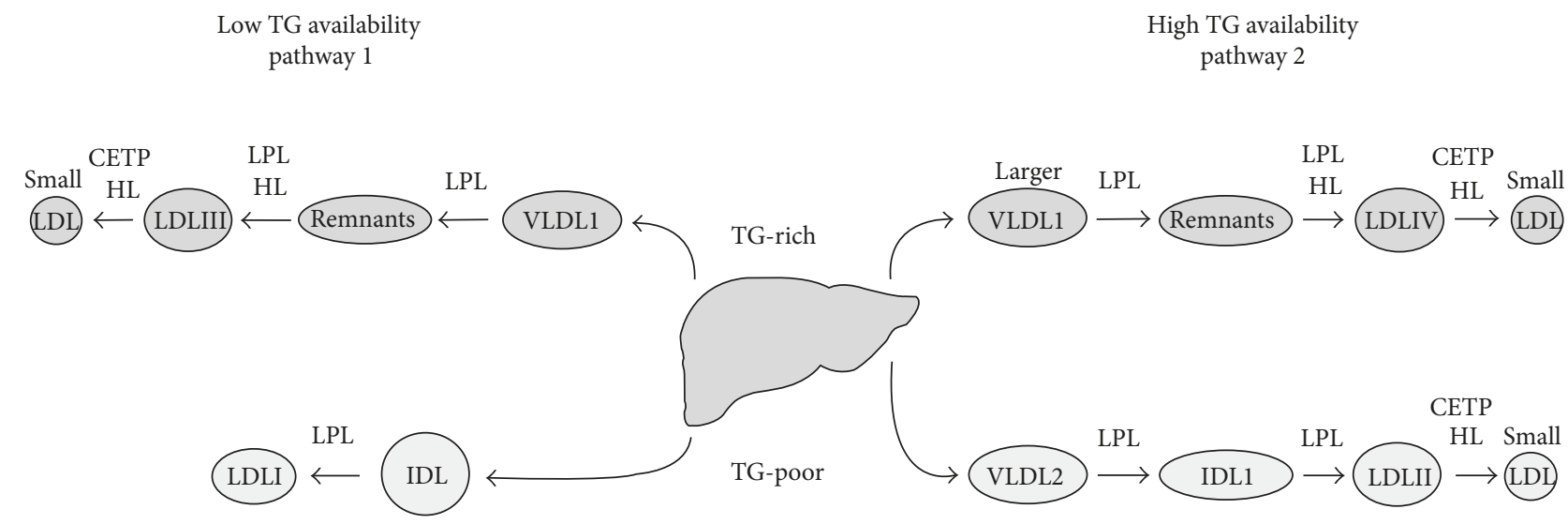

FIGURE 1: Hypothetical scheme of metabolic origins of LDL subclasses. Two metabolic pathways exist for the production of LDL particles from the precursors secreted by the liver. In case of low TG availability, the liver secretes mostly VLDL1 and IDL as TG-rich and TG-poor lipoprotein particles. These can be modified by LPL and HL to generate LDLI and III particles. In case of high TG availability, a distinct pattern of LDL precursors is secreted, including larger VLDL1 and VLDL2. After these modifications by LPL and HL, they give rise to LDLII and IV particles. After TG transfer to the LDL particles by CETP, they can be further delipidated by HL resulting in the formation of smaller LDL particles. TG: triglycerides; LPL: lipoprotein lipase; HL: hepatic lipase; CETP: cholesterol ester transfer protein.

available on the comparability of the LDL subfraction analysis methods in terms of predicting CVD outcomes [46]. Therefore, more studies are needed to develop a standard analytical procedure.

\section{Origins of LDL Subclasses}

The exact origins of LDL subclasses remain to be elucidated. Berneis et al. proposed the existence of two pathways dependent on hepatic triglyceride (TG) availability [44]. Two types of precursor lipoproteins (Lp) are secreted from the liver, containing TG-rich or TG-poor apolipoprotein B
(apoB). When the TG availability is low, VLDL1 (TG-rich Lp) and IDL2 (TG-poor Lp) are secreted. If the TG availability is high, larger particles are secreted, such as larger VLDL1 (TG-rich Lp) and VLDL2 (TG-poor Lp). TG-poor Lp is a precursor for larger LDL subclasses (LDL I and LDL II), whereas TG-rich Lp is converted into sdLDL subclasses (LDL III and LDL IV) after delipidation by lipoprotein lipase (LPL) and hepatic lipase (HL). Cholesteryl ester transfer protein (CETP) can transfer TG to sdLDL particles that will be further delipidated by $\mathrm{HL}$, resulting in the generation of smaller particles (Figure 1) [3, 44]. This theory advocates the distinct metabolic pathway for sdLDL from liver- 
secreted precursors and is supported by the results of an interventional human study that demonstrated an inverse correlation between LDL I and LDL III and between LDL II and LDL IV [44, 47]. As a consequence of step-wise modification, sdLDL particles have altered chemical contents, containing decreased amounts of phospholipids (as measured based on apolipoprotein B content), as well as free cholesterol and cholesterol ester, while TG contents remain unaltered [48].

Recent studies suggest that sdLDL can have multiple origins, at least in patients with metabolic disorders. The results of LDL subfraction analysis on days 0 to 7 after apheresis in patients with familial hypercholesterolemia demonstrated that sdLDL rebound dynamics could be best explained by the model, combining the direct pathway and delipidation of lbLDL [49]. The regulation of sdLDL production is likely to be dependent on the current metabolic status. The regulatory role of apoE and apoC-III lipoproteins in the apoB metabolism was studied in a recent work on healthy subjects and patients with hypertriglyceridemia [50]. When plasma TG levels were normal, the liver secreted primarily apoE-containing TGrich VLDL that was rapidly removed from the circulation. In hypertriglyceridemia, however, the balance was shifted towards apoC-III-containing TG-rich lipoproteins that had longer circulation times and were converted into sdLDL. Clearance of apoE-containing lipoproteins was also reduced. As a result, high rate of sdLDL formation and reduced clearance led to the development of phenotype pattern B with elevated sdLDL levels. These observations highlight the importance of controlling hypertriglyceridemia for reduction of CVD risk. Numerous studies have been conducted to evaluate the effects of lifestyle and dietary changes on TG and sdLDL production and are reviewed elsewhere [51]. Some dietary components, such as omega-3 polyunsaturated fatty acids, were demonstrated to have beneficial effects $[51,52]$.

LDL particles can be modified by CETP, which is responsible for the exchange of TG and cholesteryl ester between LDL and VLDL and/or HDL and HL. This leads to the production of smaller sdLDL particles. Correspondingly, inhibition of CETP could reduce the sdLDL fraction in individuals with low HDL-C and in healthy premenopausal women $[53,54]$.

Genetic factors influencing sdLDL production have been studied in recently performed genome-wide association studies (GWAS). It was found that a single nucleotide polymorphism (SNP) in the promoter region of sortilin, a sorting receptor involved in the hepatic release of VLDL, results in alterations in hepatic sortilin synthesis and has an influence on the lipoprotein profile. Very small LDL fraction was increased by $20 \%$ in major allele homozygotes as compared to minor allele homozygotes [55]. Other SNPs associated with altered lipoprotein metabolism have been reported in different loci, including CETP, LPL, LIPC, GALNT2, MLXIPL, APOA1/A5, and PCSK7 [40, 56]. Therefore, sdLDL metabolism is dependent on genetic factors that might be considered for the development of novel therapeutic strategies.

\section{Atherogenic Modifications of sdLDL}

The circulation time of sdLDL is longer than that of large LDL particles that are cleared from the bloodstream through the interaction with the LDL receptor [57, 58]. Lipid trapping and accumulation by foam cells in the arterial wall are the key processes that lead to the development and growth of the atherosclerotic plaque. LDL particles are the main source of cholesterol stored in the plaques and their atherogenic properties have been extensively studied. It was demonstrated that native LDL does not cause lipid accumulation in cultured cells, whereas modified particles, such as oxidized, desialylated, glycated, and electronegative LDL, are highly atherogenic $[9,59]$. Modified forms of LDL also possess proinflammatory properties and are prone to aggregation and formation of complexes that further increase their atherogenicity.

Oxidation in blood plasma is one of the first atherogenic modifications of LDL particles that have been proposed $[9,60]$. Oxidation results in the generation of oxidationspecific epitopes on the LDL particles that induce the immune response and inflammation. Oxidized LDL is recognized by a number of receptors, including CD36 and TLR- 4 [61]. Increased susceptibility of sdLDL to oxidation can be explained by its lipid composition [48]. Moreover, sdLDL particles contain less antioxidative vitamins and are therefore more susceptible to oxidation than larger forms of lipoproteins [62].

Enrichment of lipoprotein-associated phospholipase A2 (Lp-PLA2) in LDL particles is known to be associated with cardiovascular disease. High PLA2 contents were described in electronegative LDL and also in advanced atherosclerotic plaques. Inside the lipoprotein particle, this enzyme cleaves oxidized phospholipids, releasing proinflammatory products and further increasing its atherogenicity [63].

Another atherogenic modification of LDL is desialylation, which is performed in blood plasma by trans-sialidase that plays an important role in the metabolism of glycoconjugates [64]. Trans-sialidase transfers the sialic acid moiety from the LDL particle to various acceptors such as plasma proteins, neutral sphingolipids, or gangliosides. It was demonstrated that incubation of purified LDL with blood plasma for several hours leads to a gradual desialylation of the particles [64]. sdLDL have a decreased sialic acid content in comparison to lbLDL in subjects with phenotype pattern B $[6,65]$. Desialylation apparently increases the affinity of the sdLDL particles to proteoglycans in the arterial wall. As a result, desialylated sdLDL has a prolonged residence time in the subendothelial space where it can contribute to the lipid storage and atherosclerosis plaque development [66].

ApoB lipoprotein was shown to be preferentially glycated in sdLDL particles as compared to lbLDL both in vitro and in vivo $[67,68]$, and the level of glycated apoB inversely correlated by particle size measured by NMR [69].

The origins of the elevated electronegative LDL (LDL(-)) levels in the plasma of atherosclerotic patients are not completely understood. Several mechanisms have been proposed, including oxidation, modification of the protein component, and binding to proteoglycans [70]. The relationship 
TABLE 2: Comparison of characteristics of sdLDL, LDL(-), and desialylated LDL.

\begin{tabular}{|c|c|c|c|}
\hline & sdLDL & LDL( $(-)$ & Desialylated LDL \\
\hline Size and density & Small dense & Small & Small dense \\
\hline Aggregation/self-association & \multicolumn{3}{|c|}{ Increased* ability to self-associate } \\
\hline Negative charge & \multicolumn{3}{|c|}{ Increased negative charge } \\
\hline & \multicolumn{3}{|c|}{ Decreased sialic acid contents } \\
\hline Chemicl comnocition & \multicolumn{3}{|c|}{ Decreased cholesteryl esters } \\
\hline 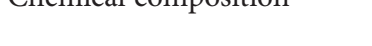 & \multicolumn{3}{|c|}{ Decreased phospholipids } \\
\hline & \multicolumn{3}{|c|}{ Increased protein/lipid ratio } \\
\hline Amino group modification & \multicolumn{3}{|c|}{ Amino group modification present } \\
\hline Oxidation & \multicolumn{3}{|c|}{ Increased oxidizability, reduced amount of antioxidants in the particles } \\
\hline Atherogenicity & \multicolumn{3}{|c|}{ Increased atherogenicity } \\
\hline
\end{tabular}

${ }^{*}$ In comparison to native (nonmodified) LDL.

of LDL(-) to sdLDL was a subject of several studies. It has been demonstrated that LDL(-) from plasma of healthy individuals was predominant in the dense subfraction, while most of the LDL(-) from patients with hypercholesterolemia was found in the light LDL fractions [71]. LDL(-) was increased in the plasma of patients with high coronary heart disease risk [72]. Another study described a bimodal distribution, with LDL(-) present in both dense and light LDL fractions [73]. It has been shown, however, that the increase in the LDL(-) production was closely related to the increase in the oxidized LDL and sdLDL levels [74].

Efforts were made to detect naturally occurring modified LDL forms in human plasma. Elevated levels of Lp(a) could be selectively detected by immunoassays developed and optimized for that purpose [75]. Although oxidized LDL could not be readily isolated, other types of modified LDL have been purified, such as desialylated LDL and LDL(-). The former could be analyzed in human serum using a lectin-sorbent assay [76] and the latter by methods sensitive to the electric charge of the particles, such as ion-exchange chromatography [77] and capillary isotachophoresis [78]. The sialic acid content of isolated $\mathrm{LDL}(-)$ particles was 1.7-fold and 3-fold lower in healthy subjects and atherosclerosis patients, respectively, as compared to native LDL [79]. On the other hand, desialylated LDL was enriched in LDL(-) [80]. These observations suggest that desialylated and electronegative LDL subfractions might be similar or even identical (Table 2). Moreover, both desialylated and $\mathrm{LDL}(-)$ particles are susceptible to oxidation and contain less antioxidant vitamins than native LDL. It is therefore plausible that LDL undergoes multiple modifications in the bloodstream, starting with desialylation and acquisition of the negative charge followed by oxidation and formation of highly atherogenic and proinflammatory complexes.

\section{5. sdLDL and Atherosclerotic CVD Risk}

The increased atherogenicity of sdLDL is linked to the specific biochemical and biophysical properties of these particles. The small size of the particles favours their penetration into the arterial wall where they serve as a source of cholesterol and lipid storage. Longer circulation time increases the probability of atherogenic modifications of sdLDL in the blood plasma. The specific role of sdLDL, the pathogenesis of atherosclerosis, and other diseases was the subject of numerous studies $[17,81]$.

It has been well documented that the predominance of sdLDL (phenotype pattern B) and elevated sdLDL-C are associated with CVD risk [8, 12, 44, 82, 83]. A recent study demonstrated that sdLDL-C concentrations were a better marker for assessment of coronary heart disease (CHD) than total LDL-C [84]. In another study, elevated sdLDL-C concentrations, but not total sdLDL particle concentrations, were found to be a significant marker of CHD risk in nondiabetic individuals. In this study, sdLDL particle fraction was measured by NMR and sdLDL-C was analyzed using an automated assay in a large number of patients [85]. A smaller prospective study conducted on type 2 diabetic and prediabetic patients demonstrated that sdLDL proportion (measured by GGE) was predictive of the increase of intima media thickness (IMT) and insulin resistance [86]. Increase sdLDL level together with CA-IMT are associated with traditional risk factors for CVD. Shen et al. suggest that SdLDL-C is a better lipid variable than other standard parameters in assessing the risk of CVD using CA-IMT, even after adjustment for traditional CVD risk factors such as higher age, male sex, smoking, and family history of CVD [87]. Finally, the association of sdLDL-C with CHD has been clearly demonstrated in a large prospective study conducted on 11,419 individuals using the homogeneous assay for sdLDL assessment [56]. sdLDL-C predicted the CHD risk even in patients considered to be at low cardiovascular risk based on their LDL-C values, therefore providing an additional value for the assessment of CVD risk.

The association of sdLDL with peripheral artery disease has also been studied recently. Elevated sdLDL contents were registered in patients with worse early outcome (improved walking distance and without restenosis) after balloon angioplasty [88].

Elevated levels of sdLDL were reported in many conditions linked to atherosclerosis, such as dyslipidemia, diabetes, and metabolic syndrome (MetS), as well as in a number of other disorders [89-92]. In MetS, the increased sdLDL levels had an independent predictive value for future 
cardiovascular events [93]. Noteworthy, the sdLDL-C/LDL-C ratio correlated better with various parameters associated with MetS and was suggested to be a more useful clinical indicator than absolute sdLDL-C and LDL-C levels [94]. Interestingly, sdLDL fraction was significantly increased in chronic kidney disease (CKD), and its measurement could be used for CVD risk assessment in patients with CKD [95].

\section{Effects of Statins and Other Therapies on sdLDL}

As the accumulating evidence points to the important role of sdLDL in the development of atherosclerosis and CVD, many studies focus on improving the lipid profile. The predominance of sdLDL is associated with the elevated TG and decreased HDL levels [96]. Hence, the goals of the corrective therapy include lowering the proportion of sdLDL-C and/or raising the HDL-C content. Statins are widely used in clinical practice as lipid lowering agents for treatment of dyslipidemia in atherosclerosis and related disorders. Despite the large amount of information available to date, it is not yet clear whether statins are efficient for specific lowering of sdLDL-C. The results of clinical studies are sometimes contradictory in that regard [57, 82, 97]. In some studies, statins failed to decrease the sdLDL proportion because larger LDL fractions were also decreased and the ratio of sdLDL-C versus lbLDL-C was unchanged [90]. Therefore the outcome of the statin treatment should be evaluated by the absolute changes of sdLDL concentrations and not their relative content or size distributions. Lack of standardization in LDL fractionation methods and varying clinical characteristics hinder the objective comparison of the results of clinical studies. More intervention studies are necessary to draw the conclusion on the effect of statin therapy on sdLDLC proportion and its relationship to CVD risk reduction [3].

Apart from statins, other hypolipidemic agents, such as ezetimibe and fibrates, had a beneficial effect of LDL subfractions [98]. Ezetimibe decreased the large and medium LDLs and, to a lesser extent, sdLDL particles [99]. Fibrates and niacin reduced sdLDL levels and shifted the distribution of LDL particle size towards lbLDL. Gemfibrozil lowered sdLDL fraction especially in subjects with the phenotype pattern B [100]. Fenofibrate improved the TG and HDL-C levels more efficiently than statins, and a combination therapy of fenofibrate and statins improved the lipid profile more potently than either of the medications in monotherapy. Although the pilot studies on type 2 diabetes patients failed to prove the efficacy of fenofibrate for the reduction of CHD risk, they demonstrated its beneficial effects on a number of vascular outcomes, such as retinopathy [101]. In patients with obesity, sdLDL levels can be corrected by antiobesity medications, such as orlistat and caloric restriction and changes in lifestyle $[102,103]$.

\section{Conclusion}

The results of recent studies demonstrate that LDL fractions have different atherogenicity, with sdLDL being more atherogenic than larger LDL subfractions. sdLDL is characterized by the enhanced ability to penetrate the arterial wall that makes it a potent source of cholesterol for the development of atherosclerotic plaque. Importantly, longer circulation times of sdLDL result in multiple atherogenic modifications of sdLDL particles in plasma, further increasing its atherogenicity. Study of the sdLDL role in the development of atherosclerosis and CVD is hindered by significant variations in LDL fractionation results obtained by different methods. The development of cheap, fast, and reliable method of quantitative LDL subfraction analysis in much needed, and significant progress has been done in that direction after the development of homogeneous assays. Statins and other lipid-lowering drugs were reported to have beneficial effects on LDL profile correction, but more studies are necessary to draw clear guidelines for sdLDL lowering in CVD prevention and treatment. Although many questions regarding the efficacy of sdLDL reduction in CVD risk management remain open, there is accumulating evidence that sdLDL-C proportion is a significant marker for CVD prediction in many conditions associated with dyslipidemia.

\section{Conflicts of Interest}

Authors declare no conflict of interest.

\section{Acknowledgments}

This work was supported by the Russian Foundation for Basic Research (Grant \# 15-04-09279).

\section{References}

[1] Prospective Studies Collaboration, S. Lewington, G. Whitlock et al., "Blood cholesterol and vascular mortality by age, sex, and blood pressure: a meta-analysis of individual data from 61 prospective studies with 55,000 vascular deaths," Lancet, vol. 370, no. 9602, pp. 1829-1839, 2007.

[2] E. J. Mills, B. Rachlis, P. Wu, P. J. Devereaux, P. Arora, and D. Perri, "Primary prevention of cardiovascular mortality and events with statin treatments: a network meta-analysis involving more than 65,000 patients," Journal of the American College of Cardiology, vol. 52, no. 22, pp. 1769-1781, 2008.

[3] S. Hirayama and T. Miida, "Small dense LDL: an emerging risk factor for cardiovascular disease," Clinica Chimica Acta, no. 414, pp. 215-224, 2012.

[4] C. Baigent, A. Keech, P. M. Kearney et al., "Efficacy and safety of cholesterol-lowering treatment: prospective meta-analysis of data from 90,056 participants in 14 randomised trials of statins," Lancet, vol. 366, no. 9493, pp. 1267-1278, 2005.

[5] J. Afilalo, A. A. Majdan, and M. J. Eisenberg, "Intensive statin therapy in acute coronary syndromes and stable coronary heart disease: a comparative meta-analysis of randomised controlled trials," Heart, vol. 93, no. 8, pp. 914-921, 2007.

[6] M. Rizzo and K. Berneis, "Low-density lipoprotein size and cardiovascular risk assessment," QJM : Monthly Journal of the Association of Physicians, vol. 99, no. 1, pp. 1-14, 2006.

[7] C. J. Packard, "Small dense low-density lipoprotein and its role as an independent predictor of cardiovascular disease," Current Opinion in Lipidology, vol. 17, no. 4, pp. 412-417, 2006. 
[8] R. M. Krauss, "Lipoprotein subfractions and cardiovascular disease risk," Current Opinion in Lipidology, vol. 21, no. 4, pp. 305-311, 2010.

[9] D. Steinberg, S. Parthasarathy, T. E. Carew, J. C. Khoo, and J. L. Witztum, "Beyond cholesterol. Modifications of low-density lipoprotein that increase its atherogenicity," The New England Journal of Medicine, vol. 320, no. 14, pp. 915-924, 1989.

[10] A. N. Orekhov, V. V. Tertov, and D. N. Mukhin, "Desialylated low density lipoprotein-naturally occurring modified lipoprotein with atherogenic potency," Atherosclerosis, vol. 86, no. 2-3, pp. 153-161, 1991.

[11] M. R. Diffenderfer and E. J. Schaefer, "The composition and metabolism of large and small LDL," Current Opinion in Lipidology, vol. 25, no. 3, pp. 221-226, 2014.

[12] M. A. Austin, J. L. Breslow, C. H. Hennekens, J. E. Buring, W. C. Willett, and R. M. Krauss, "Low-density lipoprotein subclass patterns and risk of myocardial infarction," Journal of the American Medical Association, vol. 260, no. 13, pp. 1917-1921, 1988.

[13] M. A. Austin, J. D. Brunzell, W. L. Fitch, and R. M. Krauss, "Inheritance of low density lipoprotein subclass patterns in familial combined hyperlipidemia," Arteriosclerosis, vol. 10, no. 4, pp. 520-530, 1990.

[14] M. A. Austin, H. Horowitz, E. Wijsman, R. M. Krauss, and J. Brunzell, "Bimodality of plasma apolipoprotein B levels in familial combined hyperlipidemia," Atherosclerosis, vol. 92, no. 1, pp. 67-77, 1992.

[15] A. F. Ayyobi, S. H. McGladdery, M. J. McNeely, M. A. Austin, A. G. Motulsky, and J. D. Brunzell, "Small, dense LDL and elevated apolipoprotein B are the common characteristics for the three major lipid phenotypes of familial combined hyperlipidemia," Arteriosclerosis, Thrombosis, and Vascular Biology, vol. 23, no. 7, pp. 1289-1294, 2003.

[16] F. Magkos, B. S. Mohammed, and B. Mittendorfer, "Effect of obesity on the plasma lipoprotein subclass profile in normoglycemic and normolipidemic men and women," International Journal of Obesity, vol. 32, no. 11, pp. 16551664, 2008.

[17] D. Nikolic, N. Katsiki, G. Montalto, E. R. Isenovic, D. P. Mikhailidis, and M. Rizzo, "Lipoprotein subfractions in metabolic syndrome and obesity: clinical significance and therapeutic approaches," Nutrients, vol. 5, no. 3, pp. 928948, 2013.

[18] K. Berneis, C. Jeanneret, J. Muser, B. Felix, and A. R. Miserez, "Low-density lipoprotein size and subclasses are markers of clinically apparent and non-apparent atherosclerosis in type 2 diabetes," Metabolism, Clinical and Experimental, vol. 54, no. 2, pp. 227-234, 2005.

[19] R. Goldberg, M. Temprosa, J. Otvos et al., "Lifestyle and metformin treatment favorably influence lipoprotein subfraction distribution in the Diabetes Prevention Program," The Journal of Clinical Endocrinology and Metabolism, vol. 98, no. 10, pp. 3989-3998, 2013.

[20] J. D. Brunzell, A. Zambon, and S. S. Deeb, "The effect of hepatic lipase on coronary artery disease in humans is influenced by the underlying lipoprotein phenotype," Biochimica et Biophysica Acta, vol. 1821, no. 3, pp. 365372, 2012.

[21] National Cholesterol Education Program Expert Panel on Detection E, Treatment of High Blood Cholesterol in A, "Third report of the National Cholesterol Education Program
(NCEP) expert panel on detection, evaluation, and treatment of high blood cholesterol in adults (Adult Treatment Panel III) final report," Circulation, vol. 106, no. 25, pp. 31433421, 2002.

[22] G. Schmitz and E. Orsó, "Lipoprotein(a) hyperlipidemia as cardiovascular risk factor: pathophysiological aspects," Clinical Research in Cardiology Supplements, vol. 10, Supplement 1, pp. 21-25, 2015.

[23] R. M. Krauss, F. T. Lindgren, and R. M. Ray, "Interrelationships among subgroups of serum lipoproteins in normal human subjects," Clinica Chimica Acta, vol. 104, no. 3, pp. 275-290, 1980.

[24] R. J. Havel, H. A. Eder, and J. H. Bragdon, "The distribution and chemical composition of ultracentrifugally separated lipoproteins in human serum," The Journal of Clinical Investigation., vol. 34, no. 9, pp. 1345-1353, 1955.

[25] J. W. Gofman, F. T. Lindgren, and H. Elliott, "Ultracentrifugal studies of lipoproteins of human serum," The Journal of Biological Chemistry, vol. 179, no. 2, pp. 973-979, 1949.

[26] B. A. Griffin, M. J. Caslake, B. Yip, G. W. Tait, C. J. Packard, and J. Shepherd, "Rapid isolation of low density lipoprotein (LDL) subfractions from plasma by density gradient ultracentrifugation," Atherosclerosis, vol. 83, no. 1, pp. 5967, 1990.

[27] B. A. Griffin, D. J. Freeman, G. W. Tait et al., "Role of plasma triglyceride in the regulation of plasma low density lipoprotein (LDL) subfractions: contribution of small, dense LDL to coronary heart disease risk," Atherosclerosis, vol. 106, no. 2, pp. 241-253, 1994.

[28] M. S. Yee, D. V. Pavitt, T. Tan et al., "Lipoprotein separation in a novel iodixanol density gradient, for composition, density, and phenotype analysis," Journal of Lipid Research, vol. 49, no. 6, pp. 1364-1371, 2008.

[29] I. G. Davies, J. M. Graham, and B. A. Griffin, "Rapid separation of LDL subclasses by iodixanol gradient ultracentrifugation," Clinical Chemistry, vol. 49, no. 11, pp. 1865-1872, 2003.

[30] W. Ensign, N. Hill, and C. B. Heward, "Disparate LDL phenotypic classification among 4 different methods assessing LDL particle characteristics," Clinical Chemistry, vol. 52, no. 9, pp. 1722-1727, 2006.

[31] P. T. Williams, K. M. Vranizan, and R. M. Krauss, "Correlations of plasma lipoproteins with LDL subfractions by particle size in men and women," Journal of Lipid Research, vol. 33, no. 5, pp. 765-774, 1992.

[32] D. M. Hoefner, S. D. Hodel, J. F. O'Brien et al., "Development of a rapid, quantitative method for LDL subfractionation with use of the Quantimetrix Lipoprint LDL System," Clinical Chemistry, vol. 47, no. 2, pp. 266-274, 2001.

[33] C. Banuls, L. Bellod, A. Jover et al., "Comparability of two different polyacrylamide gel electrophoresis methods for the classification of LDL pattern type," Clinica Chimica Acta, vol. 413, no. 1-2, pp. 251-257, 2012.

[34] J. D. Otvos, E. J. Jeyarajah, D. W. Bennett, and R. M. Krauss, "Development of a proton nuclear magnetic resonance spectroscopic method for determining plasma lipoprotein concentrations and subspecies distributions from a single, rapid measurement," Clinical Chemistry, vol. 38, no. 9, pp. 1632-1638, 1992.

[35] D. R. Witte, M. R. Taskinen, H. Perttunen-Nio, A. Van Tol, S. Livingstone, and H. M. Colhoun, "Study of 
agreement between LDL size as measured by nuclear magnetic resonance and gradient gel electrophoresis," Journal of Lipid Research, vol. 45, no. 6, pp. 1069-1076, 2004.

[36] M. Okazaki, S. Usui, M. Ishigami et al., "Identification of unique lipoprotein subclasses for visceral obesity by component analysis of cholesterol profile in high-performance liquid chromatography," Arteriosclerosis, Thrombosis, and Vascular Biology, vol. 25, no. 3, pp. 578-584, 2005.

[37] D. O'Neal, P. Harrip, G. Dragicevic, D. Rae, and J. D. Best, "A comparison of LDL size determination using gradient gel electrophoresis and light-scattering methods," Journal of Lipid Research, vol. 39, no. 10, pp. 2086-2090, 1998.

[38] T. Sakurai, S. Trirongjitmoah, Y. Nishibata et al., "Measurement of lipoprotein particle sizes using dynamic light scattering," Annals of Clinical Biochemistry, vol. 47, Part 5, pp. 476-481, 2010.

[39] M. P. Caulfield, S. Li, G. Lee et al., "Direct determination of lipoprotein particle sizes and concentrations by ion mobility analysis," Clinical Chemistry, vol. 54, no. 8, pp. 1307-1316, 2008.

[40] K. Musunuru, M. Orho-Melander, M. P. Caulfield et al., "Ion mobility analysis of lipoprotein subfractions identifies three independent axes of cardiovascular risk," Arteriosclerosis, Thrombosis, and Vascular Biology, vol. 29, no. 11, pp. 1975-1980, 2009.

[41] T. Hirano, Y. Ito, H. Saegusa, and G. Yoshino, "A novel and simple method for quantification of small, dense LDL," Journal of Lipid Research, vol. 44, no. 11, pp. 2193-2201, 2003.

[42] Y. Ito, M. Fujimura, M. Ohta, and T. Hirano, "Development of a homogeneous assay for measurement of small dense LDL cholesterol," Clinical Chemistry, vol. 57, no. 1, pp. 5765,2011

[43] J. J. Albers, H. Kennedy, and S. M. Marcovina, "Evaluation of a new homogenous method for detection of small dense LDL cholesterol: comparison with the LDL cholesterol profile obtained by density gradient ultracentrifugation," Clinica Chimica Acta, vol. 412, no. 7-8, pp. 556-561, 2011.

[44] K. K. Berneis and R. M. Krauss, "Metabolic origins and clinical significance of LDL heterogeneity," Journal of Lipid Research, vol. 43, no. 9, pp. 1363-1379, 2002.

[45] R. M. Krauss and D. J. Burke, "Identification of multiple subclasses of plasma low density lipoproteins in normal humans," Journal of Lipid Research, vol. 23, no. 1, pp. 97-104, 1982.

[46] M. Chung, A. H. Lichtenstein, S. Ip, J. Lau, and E. M. Balk, "Comparability of methods for LDL subfraction determination: a systematic review," Atherosclerosis, vol. 205, no. 2, pp. 342-348, 2009.

[47] R. M. Krauss, P. T. Williams, F. T. Lindgren, and P. D. Wood, "Coordinate changes in levels of human serum low and high density lipoprotein subclasses in healthy men," Arteriosclerosis, vol. 8, no. 2, pp. 155-162, 1988.

[48] H. Ohmura, H. Mokuno, M. Sawano et al., "Lipid compositional differences of small, dense low-density lipoprotein particle influence its oxidative susceptibility: possible implication of increased risk of coronary artery disease in subjects with phenotype B," Metabolism, Clinical and Experimental, vol. 51, no. 9, pp. 1081-1087, 2002.

[49] H. C. Geiss, S. Bremer, P. H. Barrett, C. Otto, and K. G. Parhofer, "In vivo metabolism of LDL subfractions in patients with heterozygous $\mathrm{FH}$ on statin therapy: rebound analysis of LDL subfractions after LDL apheresis," Journal of Lipid Research, vol. 45, no. 8, pp. 1459-1467, 2004.

[50] C. Zheng, C. Khoo, J. Furtado, and F. M. Sacks, "Apolipoprotein C-III and the metabolic basis for hypertriglyceridemia and the dense low-density lipoprotein phenotype," Circulation, vol. 121, no. 15, pp. 1722-1734, 2010.

[51] T. A. Jacobson, M. Miller, and E. J. Schaefer, "Hypertriglyceridemia and cardiovascular risk reduction," Clinical Therapeutics, vol. 29, no. 5, pp. 763-777, 2007.

[52] A. Pirillo and A. L. Catapano, "Omega-3 polyunsaturated fatty acids in the treatment of hypertriglyceridaemia," International Journal of Cardiology, vol. 170, 2 Supplement 1, pp. S16-S20, 2013.

[53] M. E. Brousseau, E. J. Schaefer, M. L. Wolfe et al., "Effects of an inhibitor of cholesteryl ester transfer protein on HDL cholesterol," The New England Journal of Medicine, vol. 350, no. 15, pp. 1505-1515, 2004.

[54] R. M. Krauss, K. Wojnooski, J. Orr et al., "Changes in lipoprotein subfraction concentration and composition in healthy individuals treated with the CETP inhibitor anacetrapib," Journal of Lipid Research, vol. 53, no. 3, pp. 540-547, 2012.

[55] K. Musunuru, A. Strong, M. Frank-Kamenetsky et al., "From noncoding variant to phenotype via SORT1 at the $1 \mathrm{p} 13$ cholesterol locus," Nature, vol. 466, no. 7307, pp. 714-719, 2010.

[56] R. C. Hoogeveen, J. W. Gaubatz, W. Sun et al., "Small dense low-density lipoprotein-cholesterol concentrations predict risk for coronary heart disease: the Atherosclerosis Risk in Communities (ARIC) study," Arteriosclerosis, Thrombosis, and Vascular Biology, vol. 34, no. 5, pp. 1069-1077, 2014

[57] C. Packard, M. Caslake, and J. Shepherd, "The role of small, dense low density lipoprotein (LDL): a new look," International Journal of Cardiology, vol. 74, Supplement 1, pp. S17-S22, 2000.

[58] B. A. Griffin, "Lipoprotein atherogenicity: an overview of current mechanisms," The Proceedings of the Nutrition Society, vol. 58, no. 1, pp. 163-169, 1999.

[59] O. Jaakkola, T. Solakivi, V. V. Tertov, A. N. Orekhov, T. A. Miettinen, and T. Nikkari, "Characteristics of low-density lipoprotein subfractions from patients with coronary artery disease," Coronary Artery Disease, vol. 4, no. 4, pp. 379385, 1993.

[60] D. Steinberg and J. L. Witztum, "Oxidized low-density lipoprotein and atherosclerosis," Arteriosclerosis, Thrombosis, and Vascular Biology, vol. 30, no. 12, pp. 2311-2316, 2010.

[61] Y. I. Miller, S. H. Choi, P. Wiesner et al., "Oxidation-specific epitopes are danger-associated molecular patterns recognized by pattern recognition receptors of innate immunity," Circulation Research, vol. 108, no. 2, pp. 235-248, 2011.

[62] D. L. Tribble, M. Rizzo, A. Chait, D. M. Lewis, P. J. Blanche, and R. M. Krauss, "Enhanced oxidative susceptibility and reduced antioxidant content of metabolic precursors of small, dense low-density lipoproteins," The American Journal of Medicine, vol. 110, no. 2, pp. 103-110, 2001.

[63] C. H. Macphee, J. J. Nelson, and A. Zalewski, "Lipoproteinassociated phospholipase A2 as a target of therapy," Current Opinion in Lipidology, vol. 16, no. 4, pp. 442-446, 2005.

[64] V. V. Tertov, V. V. Kaplun, I. A. Sobenin, E. Y. Boytsova, N. V. Bovin, and A. N. Orekhov, "Human plasma transsialidase causes atherogenic modification of low density lipoprotein," Atherosclerosis, vol. 159, no. 1, pp. 103-115, 2001. 
[65] M. La Belle and R. M. Krauss, "Differences in carbohydrate content of low density lipoproteins associated with low density lipoprotein subclass patterns," Journal of Lipid Research, vol. 31, no. 9, pp. 1577-1588, 1990.

[66] V. Anber, B. A. Griffin, M. McConnell, C. J. Packard, and J. Shepherd, "Influence of plasma lipid and LDL-subfraction profile on the interaction between low density lipoprotein with human arterial wall proteoglycans," Atherosclerosis, vol. 124, no. 2, pp. 261-271, 1996.

[67] N. Younis, V. Charlton-Menys, R. Sharma, H. Soran, and P. N. Durrington, "Glycation of LDL in non-diabetic people: small dense LDL is preferentially glycated both in vivo and in vitro," Atherosclerosis, vol. 202, no. 1, pp. 162-168, 2009.

[68] H. Soran and P. N. Durrington, "Susceptibility of LDL and its subfractions to glycation," Current Opinion in Lipidology, vol. 22, no. 4, pp. 254-261, 2011.

[69] H. Matsui, K. Okumura, Y. Toki, and T. Hayakawa, "Lowdensity lipoprotein particle size as an independent predictor of glycated low-density lipoprotein level," Diabetes Care, vol. 22, no. 7, pp. 1220-1221, 1999.

[70] A. P. Mello, I. T. da Silva, D. S. Abdalla, and N. R. Damasceno, "Electronegative low-density lipoprotein: origin and impact on health and disease," Atherosclerosis, vol. 215, no. 2, pp. 257-265, 2011.

[71] J. L. Sanchez-Quesada, S. Benitez, C. Otal, M. Franco, F. Blanco-Vaca, and J. Ordonez-Llanos, "Density distribution of electronegative LDL in normolipemic and hyperlipemic subjects," Journal of Lipid Research, vol. 43, no. 5, pp. 699705, 2002.

[72] J. L. Sanchez-Quesada, A. Perez, A. Caixas et al., "Effect of glycemic optimization on electronegative low-density lipoprotein in diabetes: relation to nonenzymatic glycosylation and oxidative modification," The Journal of Clinical Endocrinology and Metabolism, vol. 86, no. 7, pp. 32433249, 2001.

[73] B. Chappey, I. Myara, M. O. Benoit, C. Maziere, J. C. Maziere, and N. Moatti, "Characteristics of ten charge-differing subfractions isolated from human native low-density lipoproteins (LDL). No evidence of peroxidative modifications," Biochimica et Biophysica Acta, vol. 1259, no. 3, pp. 261270, 1995.

[74] G. Hasegawa, S. Kajiyama, T. Tanaka et al., "The alphaglucosidase inhibitor acarbose reduces the net electronegative charge of low-density lipoprotein in patients with newly diagnosed type 2 diabetes," Clinica Chimica Acta, vol. 390, no. 1-2, pp. 110-114, 2008.

[75] E. J. Schaefer, F. Tsunoda, M. Diffenderfer, E. Polisecki, N. Thai, and B. Asztalos, "The measurement of lipids, lipoproteins, apolipoproteins, fatty acids, and sterols, and next generation sequencing for the diagnosis and treatment of lipid disorders," in Endotext [Internet], L. J. De Groot, G. Chrousos, K. Dungan, K. R. Feingold, A. Grossman, J. M. Hershman, C. Koch, M. Korbonits, R. McLachlan, M. New, J. Purnell, R. Rebar, F. Singer and A. Vinik, Eds., MDText.com, Inc., South Dartmouth (MA), 2016.

[76] V. V. Tertov, I. A. Sobenin, and A. N. Orekhov, "Modified (desialylated) low-density lipoprotein measured in serum by lectin-sorbent assay," Clinical Chemistry, vol. 41, no. 7, pp. 1018-1021, 1995.

[77] P. Avogaro, G. B. Bon, and G. Cazzolato, "Presence of a modified low density lipoprotein in humans," Arteriosclerosis, vol. 8, no. 1, pp. 79-87, 1988.
[78] G. Schmitz, C. Mollers, and V. Richter, "Analytical capillary isotachophoresis of human serum lipoproteins," Electrophoresis, vol. 18, no. 10, pp. 1807-1813, 1997.

[79] V. V. Tertov, I. A. Sobenin, and A. N. Orekhov, "Similarity between naturally occurring modified desialylated, electronegative and aortic low density lipoprotein," Free Radical Research, vol. 25, no. 4, pp. 313-319, 1996.

[80] V. V. Tertov, G. Bittolo-Bon, I. A. Sobenin, G. Cazzolato, A. N. Orekhov, and P. Avogaro, "Naturally occurring modified low density lipoproteins are similar if not identical: more electronegative and desialylated lipoprotein subfractions," Experimental and Molecular Pathology, vol. 62, no. 3, pp. 166-172, 1995.

[81] R. Carmena, P. Duriez, and J. C. Fruchart, "Atherogenic lipoprotein particles in atherosclerosis," Circulation, vol. 109, 23 Supplement 1, pp. III2-III7, 2004.

[82] M. Rizzo and K. Berneis, "The clinical relevance of lowdensity-lipoproteins size modulation by statins," Cardiovascular Drugs and Therapy, vol. 20, no. 3, pp. 205-217, 2006.

[83] H. Arai, Y. Kokubo, M. Watanabe et al., "Small dense low-density lipoproteins cholesterol can predict incident cardiovascular disease in an urban Japanese cohort: the Suita study," Journal of Atherosclerosis and Thrombosis, vol. 20, no. 2, pp. 195-203, 2013.

[84] M. Ai, S. Otokozawa, B. F. Asztalos et al., "Small dense LDL cholesterol and coronary heart disease: results from the Framingham Offspring Study," Clinical Chemistry, vol. 56, no. 6, pp. 967-976, 2010.

[85] M. Y. Tsai, B. T. Steffen, W. Guan et al., "New automated assay of small dense low-density lipoprotein cholesterol identifies risk of coronary heart disease: the Multi-ethnic Study of Atherosclerosis," Arteriosclerosis, Thrombosis, and Vascular Biology, vol. 34, no. 1, pp. 196-201, 2014.

[86] P. A. Gerber, C. Thalhammer, C. Schmied et al., "Small, dense LDL particles predict changes in intima media thickness and insulin resistance in men with type 2 diabetes and prediabetes-a prospective cohort study," PLoS One, vol. 8, no. 8, article e72763, 2013.

[87] H. Shen, L. Xu, J. Lu et al., "Correlation between small dense low-density lipoprotein cholesterol and carotid artery intimamedia thickness in a healthy Chinese population," Lipids in Health and Disease, vol. 14, no. 1, p. 137, 2015.

[88] V. Jacomella, P. A. Gerber, K. Mosimann et al., "Small dense low density lipoprotein particles are associated with poor outcome after angioplasty in peripheral artery disease," PLoS One, vol. 9, no. 9, article e108813, 2014.

[89] T. Hirano, Y. Ito, S. Koba et al., "Clinical significance of small dense low-density lipoprotein cholesterol levels determined by the simple precipitation method," Arteriosclerosis, Thrombosis, and Vascular Biology, vol. 24, no. 3, pp. 558-563, 2004.

[90] Y. Fukushima, S. Hirayama, T. Ueno et al., "Small dense LDL cholesterol is a robust therapeutic marker of statin treatment in patients with acute coronary syndrome and metabolic syndrome," Clinica Chimica Acta, vol. 412, no. 15-16, pp. 1423-1427, 2011.

[91] F. G. Toledo, A. D. Sniderman, and D. E. Kelley, "Influence of hepatic steatosis (fatty liver) on severity and composition of dyslipidemia in type 2 diabetes," Diabetes Care, vol. 29, no. 8, pp. 1845-1850, 2006.

[92] A. M. Cali, T. L. Zern, S. E. Taksali et al., "Intrahepatic fat accumulation and alterations in lipoprotein composition in 
obese adolescents: a perfect proatherogenic state," Diabetes Care, vol. 30, no. 12, pp. 3093-3098, 2007.

[93] M. Rizzo, V. Pernice, A. Frasheri et al., "Small, dense lowdensity lipoproteins (LDL) are predictors of cardio- and cerebro-vascular events in subjects with the metabolic syndrome," Clinical Endocrinology, vol. 70, no. 6, pp. 870875, 2009.

[94] N. Satoh, H. Wada, K. Ono et al., "Small dense LDLcholesterol relative to LDL-cholesterol is a strong independent determinant of hypoadiponectinemia in metabolic syndrome," Circulation Journal: Official Journal of the Japanese Circulation Society, vol. 72, no. 6, pp. 932-939, 2008.

[95] M. Chu, A. Y. Wang, I. H. Chan, S. H. Chui, and C. W. Lam, "Serum small-dense LDL abnormalities in chronic renal disease patients," British Journal of Biomedical Science, vol. 69, no. 3, pp. 99-102, 2012.

[96] A. I. Vinik, "The metabolic basis of atherogenic dyslipidemia," Clinical Cornerstone, vol. 7, no. 2-3, pp. 27-35, 2005.

[97] S. Ip, A. H. Lichtenstein, M. Chung, J. Lau, and E. M. Balk, "Systematic review: association of low-density lipoprotein subfractions with cardiovascular outcomes," Annals of Internal Medicine, vol. 150, no. 7, pp. 474-484, 2009.

[98] M. Florentin, A. D. Tselepis, M. S. Elisaf, C. V. Rizos, D. P. Mikhailidis, and E. N. Liberopoulos, "Effect of non-statin lipid lowering and anti-obesity drugs on LDL subfractions in patients with mixed dyslipidaemia," Current Vascular Pharmacology, vol. 8, no. 6, pp. 820-830, 2010.

[99] I. Gouni-Berthold, D. P. Mikhailidis, and M. Rizzo, "Clinical benefits of ezetimibe use: is absence of proof, proof of absence?" Expert Opinion on Pharmacotherapy, vol. 13, no. 14, pp. 1985-1988, 2012.

[100] H. R. Superko, K. K. Berneis, P. T. Williams, M. Rizzo, and P. D. Wood, "Gemfibrozil reduces small low-density lipoprotein more in normolipemic subjects classified as lowdensity lipoprotein pattern B compared with pattern A," The American Journal of Cardiology, vol. 96, no. 9, pp. 1266-1272, 2005.

[101] K. McKeage and G. M. Keating, "Fenofibrate: a review of its use in dyslipidaemia," Drugs, vol. 71, no. 14, pp. 19171946, 2011.

[102] E. S. Nakou, T. D. Filippatos, M. Georgoula et al., “The effect of orlistat and ezetimibe, alone or in combination, on serum LDL and small dense LDL cholesterol levels in overweight and obese patients with hypercholesterolaemia," Current Medical Research and Opinion, vol. 24, no. 7, pp. 19191929, 2008.

[103] L. M. Morgan, B. A. Griffin, D. J. Millward et al., "Comparison of the effects of four commercially available weight-loss programmes on lipid-based cardiovascular risk factors," Public Health Nutrition, vol. 12, no. 6, pp. 799-807, 2009. 


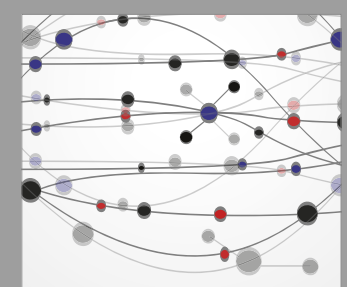

The Scientific World Journal
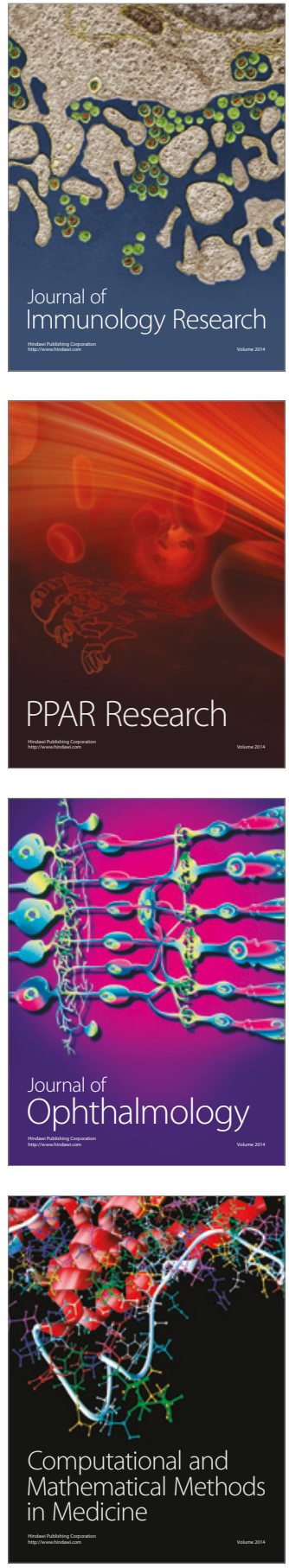

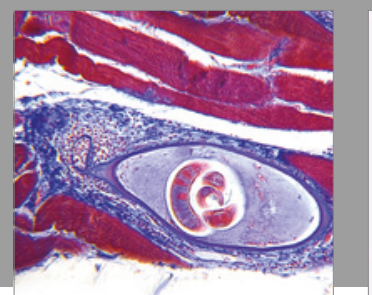

Gastroenterology Research and Practice
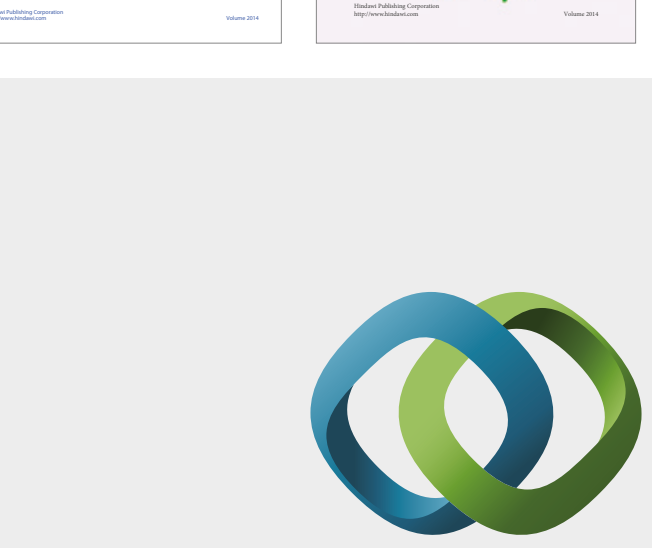

\section{Hindawi}

Submit your manuscripts at

https://www.hindawi.com
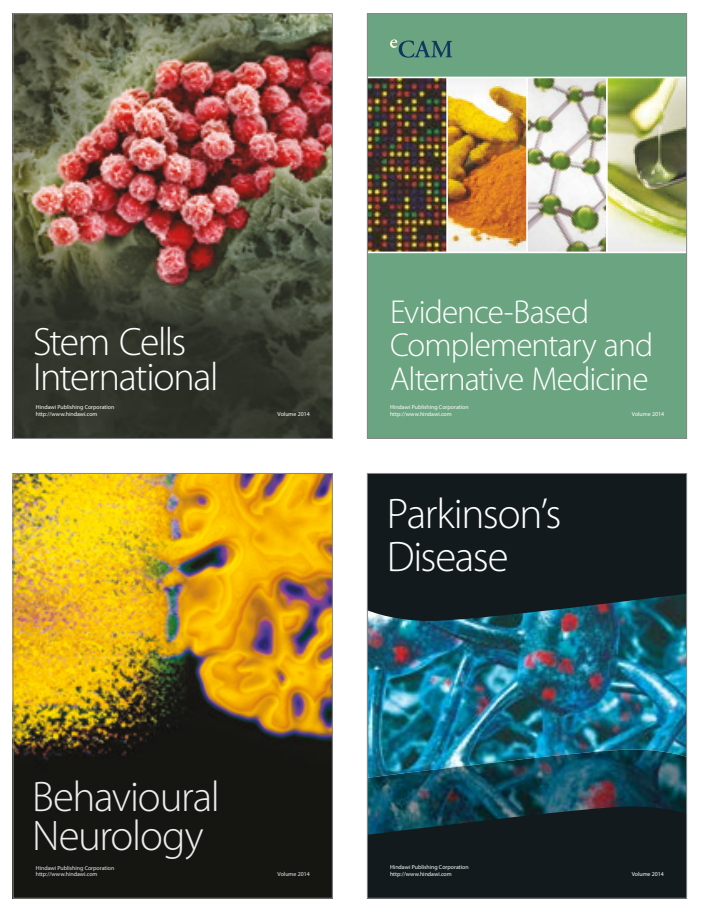
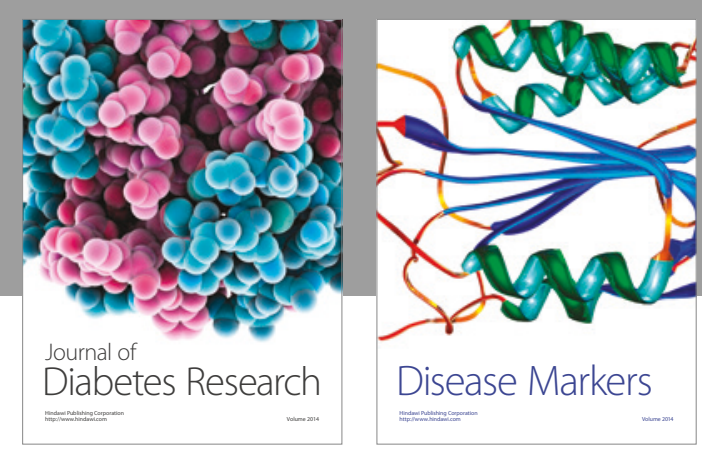

Disease Markers
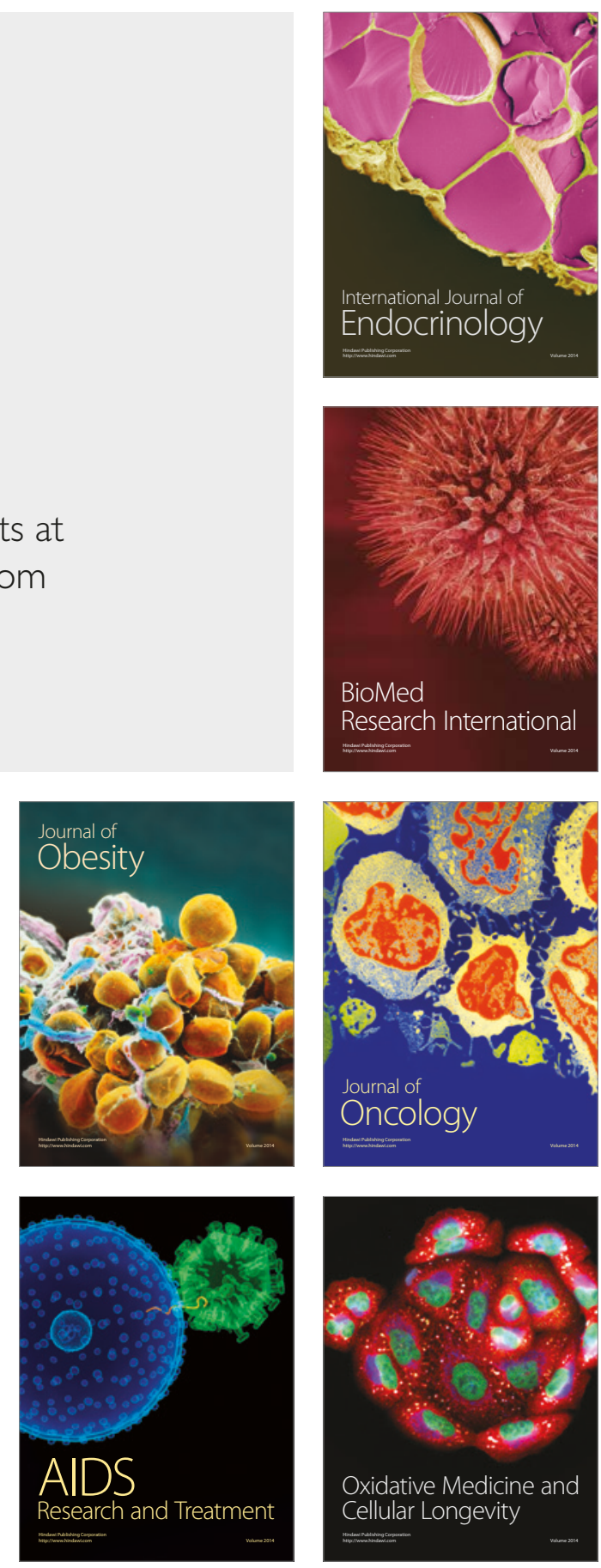